\title{
Discrete Bessel and Mathieu functions
}

\author{
Kenan Uriostegui $]^{1}$ and Kurt Bernardo Wolf \\ Instituto de Ciencias Físicas \\ Universidad Nacional Autónoma de México \\ Av. Universidad s/n, Cuernavaca, Morelos 62251, México
}

\begin{abstract}
The two-dimensional Helmholtz equation separates in elliptic coordinates based on two distinct foci, a limit case of which includes polar coordinate systems when the two foci coalesce. This equation is invariant under the Euclidean group of translations and orthogonal transformations; we replace the latter by the discrete dihedral group of $N$ discrete rotations and reflections. The separation of variables in polar and elliptic coordinates is then used to define discrete Bessel and Mathieu functions, as approximants to the well-known continuous Bessel and Mathieu functions, as $N$-point Fourier transforms approximate the Fourier transform over the circle, with integrals replaced by finite sums. We find that these 'discrete' functions approximate the numerical values of their continuous counterparts very closely and preserve some key special function relations.
\end{abstract}

\section{Introduction}

The role of the Euclidean group of translations, reflections and rotations in the determination of the coordinate systems that separate the solutions of the two-dimensional Helmholtz equation is well known from the work by Willard Miller Jr. [1, Ch. 1]. This symmetry accounts for their separability in four coordinate systems: Cartesian, polar, parabolic and elliptic. Only the elliptic system is generic; when the two foci coalesce, this system becomes the polar one with angular and radial coordinates; when one focus departs to infinity the system becomes parabolic; and when both foci do, it becomes Cartesian.

\footnotetext{
${ }^{1}$ Posgrado en Ciencias Físicas, Universidad Nacional Autónoma de México
} 
The polar decomposition was used by Biagetti et al. 2] to first introduce a discrete version of Bessel functions based on an expansion of plane waves into a finite number of polar components - that was not quite complete. This was properly completed in Ref. [3], defining discrete Bessel functions $B_{n}^{N}(\rho)$, which approximate the usual continuous Bessel functions $J_{n}(\rho)$ by replacing Fourier series over a circle $\mathcal{S}^{1}$ by the finite Fourier transform on $N$ equidistant points on that circle,

$$
\theta_{m}=2 \pi m / N, \quad m \in\{0,1, \ldots, N-1\}=: \mathcal{S}_{(N)}^{1},
$$

where $m$ is counted modulo $N$. It was found that these discrete functions approximated very closely (of the order $10^{-16}$ ) the corresponding continuous ones over a region, roughly $0 \leq n+\rho<N$.

Several authors have introduced functions that approximate the wellknown continuous Bessel functions $J_{n}(\rho)$, for the purpose of reducing computation time, or to provide new classes of solutions to difference equations that will share some of their salient properties [4, 5, 6]. Our approach follows the well known approximation afforded by the $N$-point finite Fourier transform to the integral Fourier transform over the circle. This is done for polar and elliptic coordinates, and introduces both 'discrete' Bessel and Mathieu functions. These functions, we should emphasize, differ from those proposed in the works cited above, which are also distinct in definition and purpose among themselves. By construction it will follow that under $N \rightarrow \infty$, these discrete functions become the continuous ones, although this limit requires further mathematical precision, as it may involve Gibbs-type oscillation phenomena that we cannot address here.

In Sect. 2 we present this discretization method and a resumé of the results in Ref. 3] for Bessel functions, to note that the discrete functions thus defined approximate the continuous ones remarkably well. In Sect. 3 of the present paper we apply the strategy of replacing harmonic analysis on $\mathcal{S}^{1}$ by $\mathcal{S}_{(N)}^{1}$ to define discrete approximants to the Mathieu functions of first and second kind in the elliptic coordinate system. All relations are backed by numerical verification. In the concluding Sect. 4 we provide some further connections and preliminary conclusions. 


\section{Continuous and discrete Bessel functions}

The Helmholtz equation for wavefields $f(x, y)$ of (fixed) real wavenumber $\kappa \in \mathcal{R}$, is

$$
\left(\partial_{x}^{2}+\partial_{y}^{2}+\kappa^{2}\right) f(x, y)=0
$$

with $\partial_{z} \equiv \partial / \partial_{z}$ and $(x, y) \in \mathcal{R}^{2}$. In this section we follow the well known case of polar coordinates,

$$
x=r \cos \theta, \quad y=r \sin \theta, \quad r \in[0, \infty), \quad \theta \in(-\pi, \pi]=\mathcal{S}^{1} .
$$

A key assumption is a Hilbert space structure for the solutions $f(x, y)$ by which one can write them as the two-dimensional Fourier transform,

$$
f(x, y)=\frac{1}{2 \pi} \iint_{\mathcal{R}^{2}} \mathrm{~d} \kappa_{x} \mathrm{~d} \kappa_{y} \operatorname{expi}\left(x \kappa_{x}+y \kappa_{y}\right) \tilde{f}\left(\kappa_{x}, \kappa_{y}\right) .
$$

The Helmholtz equation (2) is then correspondingly transformed to a conjugate space $\left(\kappa_{x}, \kappa_{y}\right) \in \mathcal{R}^{2}$ where it reads $\left(\kappa^{2}-\kappa_{x}^{2}-\kappa_{y}^{2}\right) \widetilde{f}\left(\kappa_{x}, \kappa_{y}\right)=0$, which we can also refer to polar coordinates $\kappa_{x}=\kappa \cos \phi, \kappa_{y}=\kappa \sin \phi$, with the surface element $\mathrm{d} \kappa_{x} \mathrm{~d} \kappa_{y}=\kappa \mathrm{d} \kappa \mathrm{d} \phi$. The solutions to the Fourier-transformed Helmholtz equation are thus reduced by a Dirac $\delta$-distributions in the radius [1, Ch. 1], as $\tilde{f}\left(\kappa_{x}, \kappa_{y}\right)=\sqrt{2 \pi} \kappa^{-1} \delta(\kappa-\widetilde{\kappa}) f_{\circ}(\phi)$, with $f_{\circ}(\phi)$ a function on the $\phi$-circle $\mathcal{S}^{1}$ of radius $\widetilde{\kappa}$, that we write again $\kappa$, understanding that it is the fixed wavenumber. The Helmholtz solutions (4) thus acquire the single-integral form

$$
f(x, y)=\frac{1}{\sqrt{ } 2 \pi} \int_{\mathcal{S}^{1}} \mathrm{~d} \phi \exp \mathrm{i} \kappa(x \cos \phi+y \sin \phi) f_{\circ}(\phi),
$$

with the Hilbert space structure based on the inner product of functions $f_{\circ}^{(1)}(\phi)$ and $f_{0}^{(2)}(\phi)$ on the circle,

$$
\left(f_{\circ}^{(1)}, f_{\circ}^{(2)}\right)_{\circ}:=\int_{\mathcal{S}^{1}} \mathrm{~d} \phi f_{\circ}^{(1)}(\phi)^{*} f_{\circ}^{(2)}(\phi) .
$$

It is here that we reduce the continuous circle Fourier transform to the $N$-point discrete Fourier transform, from $\mathcal{S}^{1}$ to $\mathcal{S}_{(N)}^{1}$, replacing integrals by summations and the continuous variable $\phi \in \mathcal{S}^{1}$ with $\phi_{m} \in \mathcal{S}_{(N)}^{1}$, as

$$
\int_{\mathcal{S}^{1}} \mathrm{~d} \phi F_{\circ}(\phi) \leftrightarrow \sum_{m \in \mathcal{S}_{(N)}^{1}} F\left(\phi_{m}\right), \quad \begin{gathered}
2 \pi \leftrightarrow N, \\
\phi_{m}=2 \pi m / N
\end{gathered}
$$


for $m \in\{0,1, \ldots, N-1\}$ counted modulo $N$; the set of $N$ discrete angles $\phi_{m}$ are thus equidistant by $2 \pi / N$. The functions $f\left(\phi_{m}\right) \equiv f_{m}$ can be interpreted as sample points of a continuous function, or as the index for the list of components of an $N$-cyclic vector. In either case, the inner product of two discrete functions $f_{n}^{(1)}$ and $f_{n}^{(2)}$ is naturally

$$
\left(f^{(1)}, f^{(2)}\right)_{(N)}:=\sum_{n=0}^{N-1} f_{n}^{(1) *} f_{n}^{(2)},
$$

and it is clear that the $N \rightarrow \infty$ limit will lead back from the discrete to the continuum, with the approximations and limits familiar from Fourier theory.

The Helmholtz equation (2) in polar coordinates, multiplied by $r^{2}$,

$$
\left(r^{2} \partial_{r}^{2}+r \partial_{r}+\partial_{\phi}^{2}+\kappa^{2}\right) f(r, \phi)=0
$$

shows that solutions can be factored into a function of the radius times a function of the angle as $f(r, \phi)=R(r) \Phi(\phi)$, while (5) implies that solutions $\Phi(\phi)$ for the angular factor will determine a corresponding radial factor $R(r)$. An orthonormal and complete set of eigenfunctions of $\partial_{\phi}^{2}$ over the circle $\phi \in \mathcal{S}^{1}$ is the set of phases $\Phi_{n}(\phi):=(2 \pi)^{-1 / 2} \exp ($ in $\phi)$, with integer $n \in$ $\{0, \pm 1, \ldots\}$, and inner products $\left(\Phi_{n}, \Phi_{n^{\prime}}\right)_{\circ}=\delta_{n, n^{\prime}}$. When the domain of these functions is restricted from $\phi \in \mathcal{S}^{1}$ to $\phi_{m} \in \mathcal{S}_{(N)}^{1}$ as in (1), we retain the subset of $N$ functions on the $N$ points in $\mathcal{S}_{(N)}^{1}$, given by

$$
\Phi_{n}^{(N)}\left(\phi_{m}\right):=\frac{1}{\sqrt{ } N} \exp \left(\mathrm{i} n \phi_{m}\right)=\frac{1}{\sqrt{ } N} \exp \left(\frac{2 \pi \mathrm{i} m n}{N}\right)=\Phi_{n \pm N}^{(N)}\left(\phi_{m}\right),
$$

labeled by the cyclic subset $n \in\{0,1, \ldots, N-1\}$, that are also orthonormal under the common inner product $(8)$ for discrete functions on $\mathcal{S}_{(N)}^{1}$, and complete:

$$
\left(\Phi_{n}^{(N)}, \Phi_{n^{\prime}}^{(N)}\right)_{(N)}=\delta_{n, n^{\prime}}, \quad \sum_{n=0}^{N-1} \Phi_{n}^{(N)}\left(\phi_{m}\right)^{*} \Phi_{n}^{(N)}\left(\phi_{m^{\prime}}\right)=\delta_{m, m^{\prime}}
$$

Returning to (5) with $(x, y)$ in the polar coordinates $(r, \theta)$ of $(3)$, and taking for $f_{\circ}\left(\phi_{m}\right)$ the basis functions $(10)$ on the discrete points of $\mathcal{S}_{(N)}^{1}$, we write the $N$ solutions to the discretized Helmholtz equation, labeled by cyclical 
$n \in\{0,1, \ldots, N-1\}$, as

$$
\begin{aligned}
f_{n}\left(r, \theta_{k}\right) & =\frac{1}{\sqrt{ } N} \sum_{m \in \mathcal{S}_{(N)}^{1}} \exp \left[\mathrm{i} \kappa r\left(\cos \theta_{k} \cos \phi_{m}+\sin \theta_{k} \sin \phi_{m}\right)\right] \Phi_{n}^{(N)}\left(\phi_{m}\right) \\
& =\frac{1}{N} \sum_{m \in \mathcal{S}_{(N)}^{1}} \exp \left[\mathrm{i} \kappa r \cos \left(\theta_{k}-\phi_{m}\right)\right] \exp \left(\mathrm{i} n \phi_{m}\right) \\
& =\frac{e^{\mathrm{i} n\left(\theta_{k}+\pi / 2\right)}}{N} \sum_{m \in \mathcal{S}_{(N)}^{1}} \exp \left(\mathrm{i} \kappa r \sin \varphi_{m}\right) \exp \left(-\mathrm{i} n \varphi_{m}\right),
\end{aligned}
$$

having replaced $\varphi_{m}:=\theta_{k}-\phi_{m}+\frac{1}{2} \pi$ in the summation over the $N$ discrete points on the circle.

Following Miller [1, p. 29], the phase in front of $[12), e^{\mathrm{i} n \theta_{n}\left(\theta_{k}+\pi / 2\right)}=$ $\mathrm{i}^{n} e^{2 \pi \mathrm{i} n k / N}=\mathrm{i}^{n} \sqrt{N} \Phi_{n}^{(N)}\left(\theta_{k}\right)$, is extracted to write the functions as

$$
f_{n}\left(r, \theta_{k}\right)=\mathrm{i}^{n} \sqrt{N} B_{n}^{(N)}(\kappa r) \Phi_{n}^{(N)}\left(\theta_{k}\right),
$$

where the radial factor $B_{n}^{(N)}(\rho), \rho:=\kappa r$, are the discrete Bessel functions. From (12) these functions are seen to be real and their parities, using coefficients $\left\{c_{n}, s_{n}\right\}:=\{1,0\}$ for $n$ even or $\{0,1\}$ for $n$ odd, can be written as

$$
\begin{aligned}
B_{n}^{(N)}(\rho) & =\frac{1}{N} \sum_{m \in \mathcal{S}_{N}^{1}} \exp \left(\mathrm{i} \rho \sin \varphi_{m}\right)\left[c_{n} \cos \left(n \varphi_{m}\right)-\mathrm{i} s_{n} \sin \left(n \varphi_{m}\right)\right] \\
& =\frac{1}{N} \sum_{m \in \mathcal{S}_{N}^{1}} \exp \left(\mathrm{i} \rho \sin \varphi_{m}\right) \times\left\{\begin{aligned}
\cos n \varphi_{m}, & n \text { even }, \\
-\mathrm{i} \sin n \varphi_{m}, & n \text { odd }
\end{aligned}\right.
\end{aligned}
$$

The distinction between even and odd cases of $n$, as done in [3], is subtle but important to obtain the correct result for all $n$ 's (cf. [2, Eq. (9)]). It results in the parity and cyclicity properties

$$
B_{n}^{(N)}(\rho)=B_{n \pm N}^{(N)}(\rho)=(-1)^{n} B_{-n}^{(N)}(\rho)=(-1)^{n} B_{n}^{(N)}(-\rho), \quad B_{n}^{(N)}(0)=\delta_{n, 0},
$$

which also hold for the continuous Bessel functions $J_{n}(\rho)$ of integer order [8].

A plane wave of wavenumber $\kappa$ along the $y$-axis in a Helmholtz medium that allows only $N$ equidistant directions of propagation on the circle, can be obtained from (14) using the completeness relation (11) to expand the 
middle term and write

$$
\begin{aligned}
\exp \left(\mathrm{i} \rho \sin \varphi_{m}\right)=B_{0}^{(N)}(\rho) & +2 \sum_{n=1}^{N-1} B_{2 n}^{(N)}(\rho) \cos \left(2 n \varphi_{m}\right) \\
& +2 \mathrm{i} \sum_{n=0}^{N-1} B_{2 n+1}^{(N)}(\rho) \sin \left((2 n+1) \varphi_{m}\right),
\end{aligned}
$$

showing how the discrete Bessel functions can take the place of the continuous ones, $c f$. [7, Eq. KU120(13)].

In Ref. [3] we proved analytically, and verified numerically, that the following expressions for the discrete Bessel functions are exact analogues of those valid for continuous Bessel functions. Corresponding to [7, WA44] for odd $N=: 2 j+1$, in Ref. [3] we proved the linear relations involving the even and odd- $n$ discrete Bessel functions,

$$
\begin{aligned}
B_{0}(\rho)+\sum_{n=1}^{j} B_{2 n}(\rho) \cos \left(2 n \varphi_{m}\right) & =\cos \left(\rho \sin \varphi_{m}\right), \\
\sum_{n=0}^{j} B_{2 n+1}(\rho) \sin \left((2 n+1) \varphi_{m}\right) & =\frac{1}{2} \sin \left(\rho \sin \varphi_{m}\right) .
\end{aligned}
$$

The quadratic formulas [9, §7.6.2, Eq. (6)] associated to the name of Graf, were shown in Ref. [10] to derive from the rotation of spherical harmonics through Wigner- $D$ functions, under contraction from the rotation to the Euclidean group. These relations, of group-theoretical origin, retain their validity under the discretization of the rotation subgroup, and lead to

$$
\sum_{n=-2 j}^{2 j} B_{n}(\rho) B_{n^{\prime}-n}\left(\rho^{\prime}\right)=B_{n^{\prime}}\left(\rho+\rho^{\prime}\right),
$$

keeping in mind the parity property (15) for the negative $n$-indices in the sum for odd $N$, addressing the vector rather than spin representations of the rotation group.

In Fig. 1 we essentially repeat the figure in Ref. [3] where we compared the discrete and continuous Bessel functions, $B_{n}^{(N)}(\rho)$ and $J_{n}(\rho)$, to support the claim that the approximation is indeed remarkable within an interval that is roughly $0 \leq n+\rho<N$. A similar set of figures is presented below for Mathiew functions.

Now, having $N$ basis functions $B_{n}^{(N)}(\rho)$, numbered by cyclic $n$ modulo $N$, it is natural to inquire whether the argument $\rho$ can or should be also 

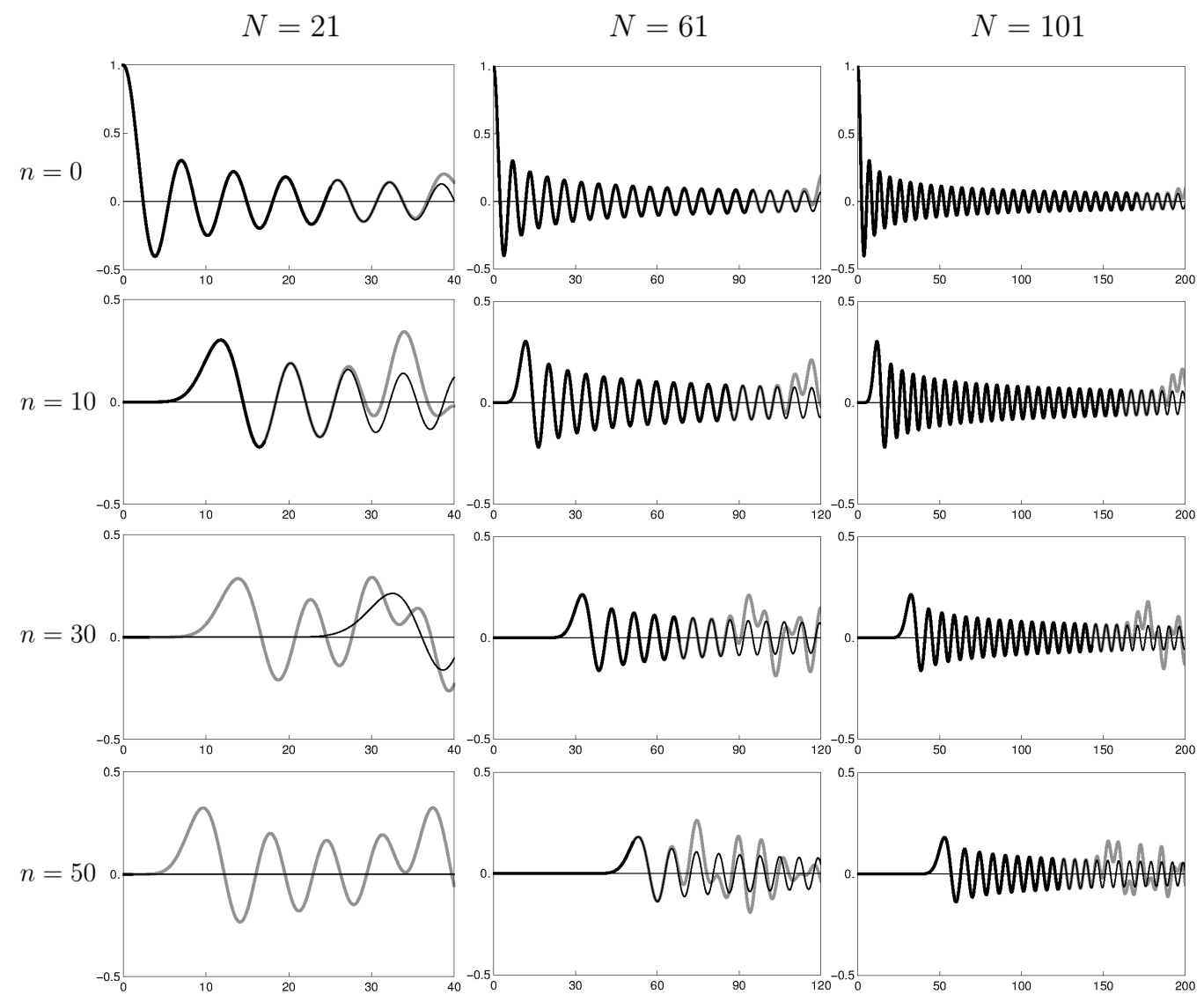

Figure 1: The 'discrete' Bessel functions $B_{n}^{(N)}(\rho)$ on continuous intervals $0 \leq \rho \leq(2 N-1)$ (gray lines), vs. the 'continuous' Bessel functions $J_{n}(\rho)$ (thin black lines), for orders $n \in$ $\{0,10,30,50\}$ and point numbers $N \in\{21,61,101\}$. Heavy black lines replace both where the 'discrete' and the 'continuous' Bessel functions differ by less than $10^{-16}$. 
discretized to the $N$ integer values $\rho_{k}=k \in\{0,1, \ldots, N-1\}$. This was done in Ref. 2] while in [3] the plot in Fig. 11 marked these points and used them to define a kernel $B_{n}^{(N)}\left(\rho_{k}\right)$ for a 'discrete Bessel transform' between two $N$-vectors of components $f_{n}$ and $\widetilde{f}_{k}$. The fact is that while the angle $\varphi$ is discretized naturally to $N$ points on the circle, the radial coordinate $\rho$ is not subject to a similarly compelling set of points, but is valid and non-cyclic over the complex $\rho$-plane. The same discretization process for the angular - but not the radial - coordinate will be applied to the Mathieu case below.

\section{Discrete Mathieu functions}

Elliptic coordinates on the plane generalize the previous polar coordinates (3); they are defined in terms of Cartesian coordinates through

$$
x=\cosh \varrho \cos \psi, \quad y=\sinh \varrho \sin \psi, \quad \varrho \in[0, \infty), \quad \psi \in(-\pi, \pi]=\mathcal{S}^{1} .
$$

where $(\varrho, \psi)$ are analogues of the previous polar coordinates $(r, \phi)$ for which we retain the names as 'radial' and 'angular' variables. For fixed $\varrho$ or for fixed $\psi$, the locus of points $(x, y) \in \mathcal{R}^{2}$ that satisfy

$$
x^{2} / \cosh ^{2} \varrho+y^{2} / \sinh ^{2} \varrho=1, \quad x^{2} / \cos ^{2} \psi-y^{2} / \sin ^{2} \psi=1,
$$

draw families of confocal ellipses or hyperbolas respectively. At $\varrho=0, \psi \in \mathcal{S}^{1}$ draws twice the line between the two foci $(x, y)=( \pm 1,0)$ for $\psi=(0, \pi)$. The major and minor semi-axes of the ellipses are cosh $\varrho$ and $\sinh \varrho$ respectively, so their eccentricities are $1 / \cosh \varrho$, that tend to circles when $\varrho \rightarrow \infty$. On the other hand, for fixed $\psi \in \mathcal{S}^{1}$ in each of the four quadrants, since $\varrho \geq 0$, only one of the four arms of the hyperbola is traversed. Thus we expect four parity cases out of the two reflections, across the $x$ and $y$ axes. Compare this with the case of polar coordinates where $r \geq 0$ but all reflection axes are equivalent, so $(-1)^{n}$ in (14) provides the two Bessel parity cases.

The Helmholtz differential equation (2), written in the elliptic coordinates (20), is clearly separable,

$$
\left[\left(\partial_{\varrho}^{2}+\kappa^{2} \cosh ^{2} \varrho\right)+\left(\partial_{\psi}^{2}-\kappa^{2} \cos ^{2} \psi\right)\right] f(\varrho, \psi)=0,
$$

so that solutions can be written in the product form $f(\varrho, \psi) \sim P(\varrho) \Psi(\psi)$. Dividing by $f$, one obtains two coupled equations in $\varrho$ and $\psi$, the latter is 


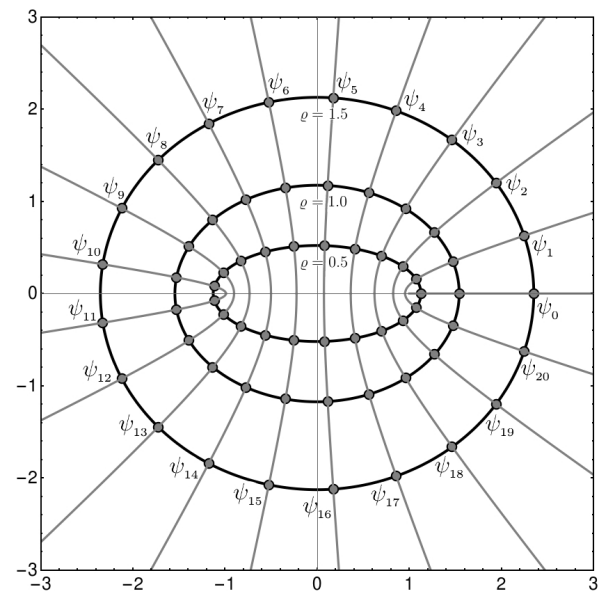

Figure 2: Set of equally-spaced discrete points on ellipses 20 of the 'angular' coordinates $\left\{\psi_{m}\right\} \in \mathcal{S}_{(N)}^{1}$ for $N=21$, and hyperbolas of the 'radial' coordinate for $\varrho \in\{0.5,1,1.5\}$.

an eigenvalue equation in the angular coordinate,

$$
\left(\partial_{\psi}^{2}-2 q \cos 2 \psi\right) \Psi(\psi, q)=\nu \Psi(\psi, q), \quad q:=\frac{1}{4} \kappa^{2},
$$

known as the Mathieu differential equation. The angular coordinate $\psi$ is periodic and a well-known solution method consists in expanding solutions of (23) in the Fourier basis $\sim \exp (\mathrm{in} \psi)$ over all integer $n$. This defines the Mathieu functions of the first kind $\operatorname{ce}_{n}(\psi, q)$ and $\operatorname{se}_{n}(\psi, q)$ with integer $n$ [11], characterized by a parity index $p \in\{0,1\}$ for even and odd cases [7, Eqs. 8.61], and distinct for even and odd indices. In a two-line expression all cases can be written as

$$
\left[\begin{array}{l}
\operatorname{ce}_{2 n+p}(\psi, q) \\
\operatorname{se}_{2 n+p}(\psi, q)
\end{array}\right]=\sum_{s=0}^{\infty}\left[\begin{array}{l}
A_{2 s+p}^{2 n+p} \cos ((2 s+p) \psi) \\
B_{2 s+p}^{2 n+p} \sin ((2 s+p) \psi)
\end{array}\right] .
$$

The parities are even $\mathrm{ce}_{n}(-\psi, q)=\mathrm{ce}_{n}(\psi, q)$, odd $\operatorname{se}_{n}(-\psi, q)=-\mathrm{se}_{n}(\psi, q)$, and $\operatorname{se}_{0}(\psi, q) \equiv 0$. The coefficients $A_{s}^{n}, B_{s}^{n}$ are found introducing this expansion into (23) to find recursion relations [7, Eqs. 8.62] that lead to efficient numerical computation. For use below, we write them using Fourier series as

$$
\left[\begin{array}{l}
A_{s}^{n} \\
B_{s}^{n}
\end{array}\right]=\frac{1}{\pi} \int_{\mathcal{S}^{1}} \mathrm{~d} \psi\left[\begin{array}{c}
\cos (s \psi) \operatorname{ce}_{n}(\psi, q) \\
\sin (s \psi) \operatorname{se}_{n}(\psi, q)
\end{array}\right],
$$

for $n \neq 0$, while $A_{0}^{n}=(2 \pi)^{-1} \int_{\mathcal{S}^{1}} \mathrm{~d} \psi \operatorname{ce}_{n}(\psi, q)$, and $B_{0}^{n} \equiv 0$. The Mathieu functions (24) are orthogonal under the inner product (6) over the circle, 
$\left(\mathrm{ce}_{m}, \mathrm{ce}_{n}\right)_{\circ}=\pi \delta_{m, n},\left(\mathrm{se}_{m}, \mathrm{se}_{n}\right)_{\circ}=\pi \delta_{m, n}$ for $n \neq 0$-zero otherwise, and $\left(\mathrm{ce}_{m}, \mathrm{se}_{n}\right)_{\circ}=0$.

We now restrict the range of the angular coordinate $\psi$ from $\mathcal{S}^{1}$ to $\mathcal{S}_{(N)}^{1}$, shown for the elliptic coordinates in Fig. 2, in correspondence with the previous discrete phase functions in the Bessel case (10), and thus defining the 'angular' discrete Mathieu functions of the first type over $\psi_{m} \in \mathcal{S}_{(N)}^{1}$ as

$$
\left[\begin{array}{l}
\operatorname{ce}_{2 n+p}^{(N)}\left(\psi_{m}, q\right) \\
\operatorname{se}_{2 n+p}^{(N)}\left(\psi_{m}, q\right)
\end{array}\right]:=\sum_{s=0}^{N-1}\left[\begin{array}{l}
a_{2 s+p}^{2 n+p} \cos \left((2 s+p) \psi_{m}\right) \\
b_{2 s+p}^{2 n+p} \sin \left((2 s+p) \psi_{m}\right)
\end{array}\right],
$$

with coefficients $a_{s}^{n}, b_{s}^{n}$. The finite $N$-point Fourier transform approximates them through the replacement (7) to the functions and coefficients $A_{s}^{n}, B_{s}^{n}$ of the continuous case in 25 , as

$$
\left[\begin{array}{l}
a_{s}^{n} \\
b_{s}^{n}
\end{array}\right]:=\frac{1}{N} \sum_{m=0}^{N-1}\left[\begin{array}{c}
\cos \left(s \psi_{m}\right) \operatorname{ce}_{n}^{(N)}\left(\psi_{m}, q\right) \\
\sin \left(s \psi_{m}\right) \operatorname{se}_{n}^{(N)}\left(\psi_{m}, q\right)
\end{array}\right] \simeq \frac{1}{2}\left[\begin{array}{c}
A_{s}^{n} \\
B_{s}^{n}
\end{array}\right]
$$

for $s \neq 0$, while $a_{0}^{n}=A_{0}^{n}, b_{0}^{n}=0$, and also $b_{s}^{0}=0$.

The last relation in (27) is an approximate equality, the validity of which is contingent upon the numerical computation and comparison between the lower- and upper-case coefficients within a range of their indices in, say, $0 \leq n, s \leq N-1$, which is reflected in turn by the discrete and continuous Mathieu functions themselves. In Fig. 3 we compare a sample of continuous angular Mathieu functions of the first kind with their discrete approximations from Eq. (26). In favor of the thus defined discrete Mathieu functions, we note that they satisfy orthogonality relations under the discrete inner product (8), namely

$$
\left(\mathrm{ce}_{n}^{(N)}, \mathrm{ce}_{n^{\prime}}^{(N)}\right)_{(N)}=\frac{1}{2} N \delta_{n, n^{\prime}}, \quad\left(\mathrm{se}_{n}^{(N)}, \mathrm{se}_{n^{\prime} \neq 0}^{(N)}\right)_{(N)}=\frac{1}{2} N \delta_{n, n^{\prime}}, \quad\left(\mathrm{ce}_{n}^{(N)}, \mathrm{se}_{n^{\prime}}^{(N)}\right)_{(N)}=0 .
$$

By construction, the parities of the discrete Mathieu functions are also even $\mathrm{ce}_{n}^{(N)}\left(-\psi_{m}, q\right)=\operatorname{ce}_{n}^{(N)}\left(\psi_{m}, q\right)$, or odd $\operatorname{se}_{n}^{(N)}\left(-\psi_{m}, q\right)=-\operatorname{se}_{n}^{(N)}\left(\psi_{m}, q\right)$.

At this point it is illuminating to inquire into the manner in which the discrete functions approximate the continuous ones. Consider for example how $\operatorname{ce}_{0}^{(N)}\left(\psi_{m}, q\right)$, whose definition (26) allows us to compute it for continuous $\psi_{m} \in \mathcal{S}^{1}$, matches $\operatorname{ce}_{0}(\psi, q)$ in the whole $\psi$ range. In Fig. 4 we do so for small $N$, noting that where the continued $\psi_{m}$ lines of the former take their values, they intersect the properly continuous line of the latter; although the two lines intersect also at other points, the two lines remain notably distinct. 

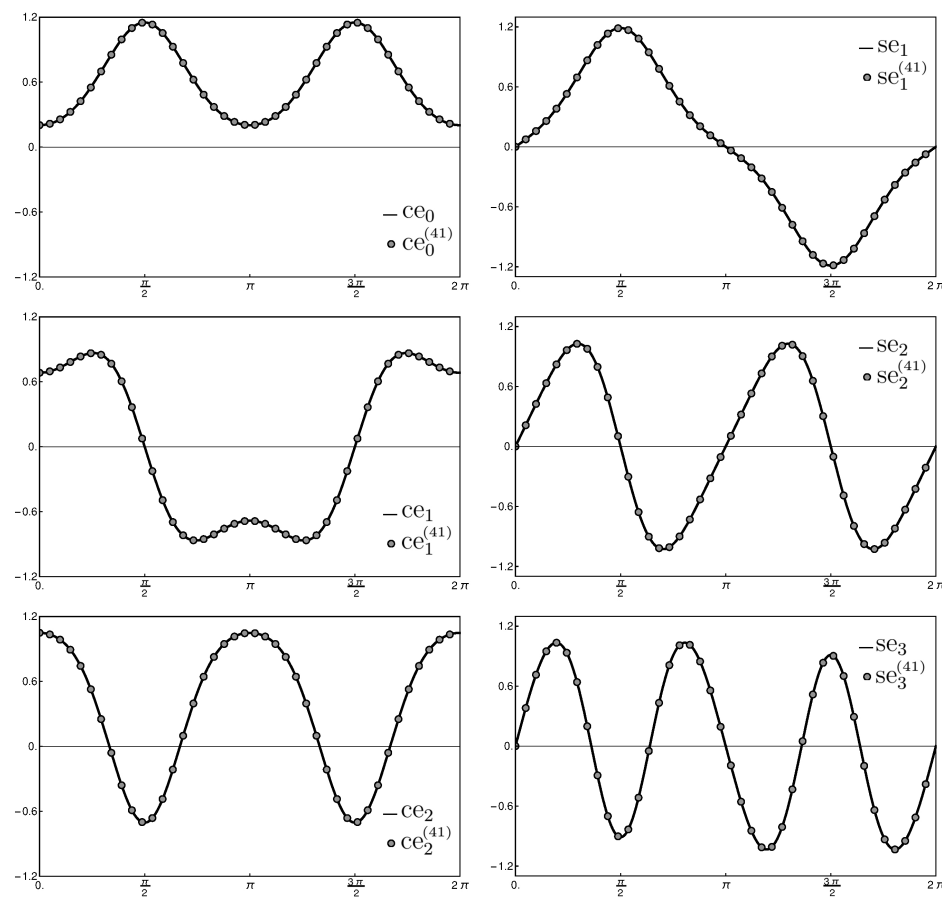

Figure 3: Discrete vs. continuous 'angular' Mathieu functions for $N=41, q=2$. The values of the discrete functions $\operatorname{ce}_{n}^{(N)}\left(\psi_{m}, q\right)$ and $\operatorname{se}_{n}^{(N)}\left(\psi_{m}, q\right)$ at $\psi_{m}, 0 \leq m \leq N-1$, are indicated by circles. The continuous Mathieu functions $\operatorname{ce}_{n}(\psi, q)$ and $\operatorname{se}_{n}(\psi, q)$ are marked by black lines in their full range $0 \leq \psi<2 \pi$. Their difference is less than $10^{-16}$ for all points $\psi_{m}$.

As the figure shows, the approximation is not valid over presumably small ranges around these intersections, but only at the prescribed $\psi_{m}=2 \pi m / N$ points. We intend to elaborate on such and similar limits elsewhere.

Proceeding now as we did in (12), but using the discrete Mathieu functions of the first kind $\mathrm{ce}_{n}^{(N)}\left(\psi_{m}, q\right)$ and $\mathrm{se}_{n}^{(N)}\left(\psi_{m}, q\right)$ in place of the plain phase functions $\Phi_{n}^{(N)}\left(\phi_{m}\right)$, we again have Helmholtz solutions $f_{n}\left(\varrho, \psi_{k}\right)$ on the plane that are characterized by parities and sub-indices $n$, whose radial factor will be the discrete 'radial' Mathieu functions of the second kind, to be indicated correspondingly as $\mathrm{Ce}_{n}^{(N)}(\varrho, q)$ and $\mathrm{Se}_{n}^{(N)}(\varrho, q)$,

$$
\begin{aligned}
{\left[\begin{array}{c}
f_{2 n+p}^{c}\left(\varrho, \psi_{k}\right) \\
f_{2 n+p+1}^{s}\left(\varrho, \psi_{k}\right)
\end{array}\right] } & =\frac{1}{N} \sum_{m=1}^{N-1}\left[\begin{array}{c}
\mathrm{ce}_{n}^{(N)}\left(\psi_{m}, q\right) \\
\operatorname{se}_{n}^{(N)}\left(\psi_{m}, q\right)
\end{array}\right] \exp \left[\mathrm{i} \kappa\left(x \cos \psi_{m}+y \sin \psi_{m}\right)\right. \\
& =:\left[\begin{array}{c}
c_{n}(q) \operatorname{Ce}_{n}^{(N)}(\varrho, q) \mathrm{ce}_{n}^{(N)}\left(\psi_{k}, q\right) \\
s_{n}(q) \operatorname{Se}_{n}^{(N)}(\varrho, q) \operatorname{se}_{n}^{(N)}\left(\psi_{k}, q\right)
\end{array}\right],
\end{aligned}
$$




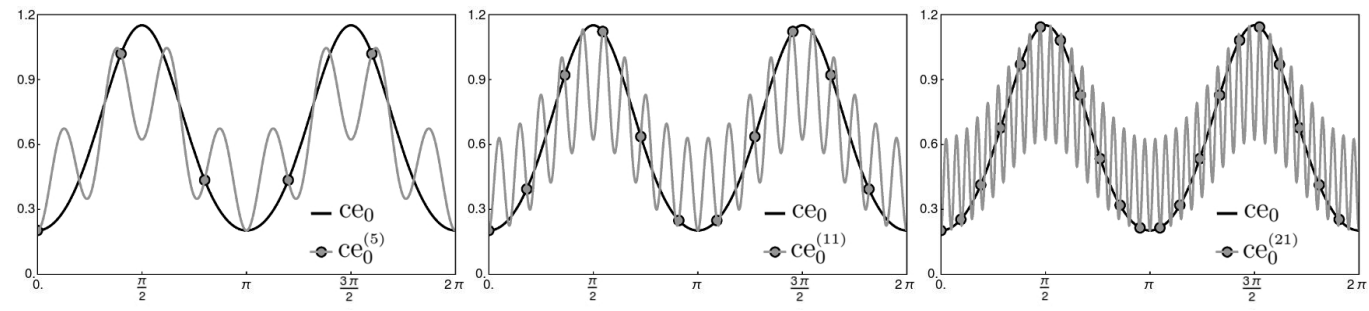

Figure 4: Comparison between the 'discrete' Mathieu functions $\operatorname{ce}_{0}^{(N)}\left(\psi_{m}, q\right)$ whose arguments are continued to $\psi_{m} \in \mathcal{S}^{1}$ (gray line) vs. the 'continuous' Mathieu function $\operatorname{ce}_{n}(\psi, q)$ (black line), for point numbers $N \in\{5,11,21\}$ and $q=2$. The discrete points $\psi_{m} \in \mathcal{S}_{(N)}^{1}$ lie at a subset of the intersections marked with circles.

where $c_{n}(q)$ and $s_{n}(q)$ are constants. Using the elliptic coordinates with a discretized angular part, $x\left(\varrho, \psi_{k}\right)=\cosh \varrho \cos \psi_{k}$ and $y\left(\varrho, \psi_{k}\right)=\sinh \varrho \sin \psi_{k}$ in (20), the phase exponent is then $\mathrm{i} \kappa=2 \mathrm{i} \sqrt{ } q$ times $x \cos \psi_{m}+y \sin \psi_{m}=$ $\cosh \varrho \cos \psi_{k} \cos \psi_{m}+\sinh \varrho \sin \psi_{k} \sin \psi_{m}$. As was done before in $(12)$ and (13), we extract the new discrete 'radial' functions using the orthogonality (28) of the previous discrete 'angular' Mathieu functions, as

$$
\begin{aligned}
{\left[\begin{array}{c}
\mathrm{Ce}_{2 n+p}^{(N)}(\varrho, q) \\
\operatorname{Se}_{2 n+p+1}^{(N)}(\varrho, q)
\end{array}\right]=} & {\left[\begin{array}{c}
1 / N c_{2 n+p}(q) \mathrm{ce}_{2 n+p}^{(N)}\left(\psi_{k}, q\right) \\
1 / N s_{2 n+p+1}(q) \operatorname{se}_{2 n+p+1}^{(N)}\left(\psi_{k}, q\right)
\end{array}\right] \sum_{m=0}^{N-1}\left[\begin{array}{c}
\operatorname{ce}_{2 n+p}^{(N)}\left(\psi_{m}, q\right) \\
\operatorname{se}_{2 n+p+1}^{(N)}\left(\psi_{m}, q\right)
\end{array}\right] } \\
& \times \exp \left[2 \mathrm{i} \sqrt{q}\left(\cosh \varrho \cos \psi_{k} \cos \psi_{m}+\sinh \varrho \sin \psi_{k} \sin \psi_{m}\right)\right] .
\end{aligned}
$$

The coefficients in front of the summation will be now determined through considering specific values for the 'angular' coordinate $\psi \leftrightarrow \psi_{m}$, comparing them with expressions of the continuous Mathieu functions of the second kind obtained from integrals that are tabulated in Ref. [7, §6.92]. There, the exponential factors appear with only a single summand in the exponent, either sine or cosine. This occurs in (31) only for $\psi_{m}=0$ or $\frac{1}{2} \pi$, although the latter is not in the set $\mathcal{S}_{(N)}^{1}$ if $\psi_{0}=0$, since $N$ was assumed to be odd.

Let us first consider the case of even parity $p=0$ and the angle $\psi=\frac{1}{2} \pi$ in (31), where the previous remark applies. Based on the close approximation between the discrete and continuous Mathieu functions, we may simply replace the latter for the former, so that the two lines in that expression read

$$
\left[\begin{array}{c}
\mathrm{Ce}_{2 n}^{(N)}(\varrho, q) \\
\operatorname{Se}_{2 n+1}^{(N)}(\varrho, q)
\end{array}\right]=\left[\begin{array}{c}
K_{2 n}^{c} \\
K_{2 n+1}^{s}
\end{array}\right] \sum_{m=0}^{N-1}\left[\begin{array}{c}
\mathrm{ce}_{2 n}^{(N)}\left(\psi_{m}, q\right) \\
\operatorname{se}_{2 n+1}^{(N)}\left(\psi_{m}, q\right)
\end{array}\right] \exp \left(2 \mathrm{i} \sqrt{q} \sinh \varrho \sin \psi_{m}\right) .
$$

When this summation formula is compared with the integral expressions 
tabulated in [7, §6.92], namely

$$
\begin{aligned}
{\left[\begin{array}{c}
\mathrm{Ce}_{2 n}(\varrho, q) \\
\operatorname{Se}_{2 n+1}(\varrho, q)
\end{array}\right]=} & {\left[\begin{array}{c}
\operatorname{ce}_{2 n}(0, q) / 2 \pi A_{0}^{2 n} \\
-\operatorname{ise}_{2 n+1}^{\prime}(0, q) / 2 \pi B_{1}^{2 n+1} \sqrt{q}
\end{array}\right] } \\
& \times \int_{\mathcal{S}^{1}} \mathrm{~d} \psi\left[\begin{array}{c}
\operatorname{ce}_{2 n}(\psi, q) \\
\operatorname{se}_{2 n+1}(\psi, q)
\end{array}\right] \exp (2 \mathrm{i} \sqrt{q} \sinh \varrho \sin \psi),
\end{aligned}
$$

we conclude that the constants in the summation (32), after identifying $2 \pi \leftrightarrow$ $N, A_{0}^{2 n}=a_{0}^{2 n}$ and $B_{1}^{2 n+1} \simeq 2 b_{1}^{2 n+1}$, are

$$
K_{2 n}^{c}=\frac{\mathrm{ce}_{2 n}(0, q)}{a_{0}^{2 n} N}, \quad K_{2 n+1}^{s}=\frac{-\mathrm{ise}_{2 n+1}^{\prime}(0, q)}{2 b_{1}^{2 n+1} N \sqrt{ } q} .
$$

where $\operatorname{se}_{n}^{\prime}(0, q):=\mathrm{d}_{n}(\psi, q) /\left.\mathrm{d} \psi\right|_{\psi=0}$. In Fig. 5 we compare a sample of the discrete and continuous 'radial' Mathieu functions, noting that the two lines are quite coincident in the range $\varrho \in[0, \pi)$, but that the discrete approximant oscillates wildly beyond $\pi$. Again, here we can only justify this statement numerically.

Next we consider the case of odd parity $p=1$ at the value $\psi=\psi_{0}=0$. The upper line in 32 reads

$$
\mathrm{Ce}_{2 n+1}^{(N)}(\varrho, q)=K_{2 n+1}^{c} \sum_{m=0}^{N-1} \operatorname{ce}_{2 n+1}^{(N)}\left(\psi_{m}, q\right) \exp \left(2 \mathrm{i} \sqrt{q} \cosh \varrho \cos \psi_{m}\right),
$$

that we compare with the integral for the continuous Mathieu functions of the second kind in [7, §6.92], namely

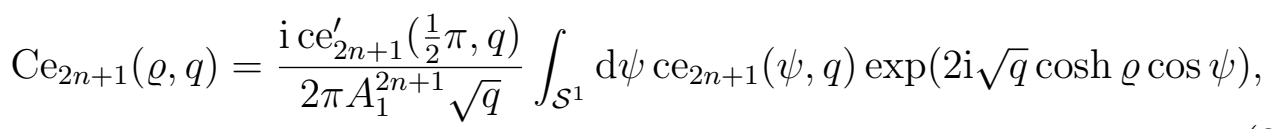

where $\operatorname{ce}_{2 n+1}^{\prime}(\psi, q)$ is the derivative of the Mathieu function. Again exploiting the correspondences (7), $2 \pi \leftrightarrow N$ and $A_{1}^{2 n+1} \simeq 2 a_{1}^{2 n+1}$, we conclude the constant in 38 to be

$$
K_{2 n+1}^{c}=\frac{\mathrm{i} \mathrm{e}_{2 n+1}^{\prime}\left(\frac{1}{2} \pi, q\right)}{2 a_{1}^{2 n+1} N \sqrt{ } q} .
$$

The remaining case to be considered is that of odd parity and even index, namely for $\mathrm{Se}_{2 n+2}^{(N)}(\varrho, q)$. This presents a problem though, because the summation 31 is identically zero for both $\psi_{k}=0$ and $\frac{1}{2} \pi$ due to the parities of 

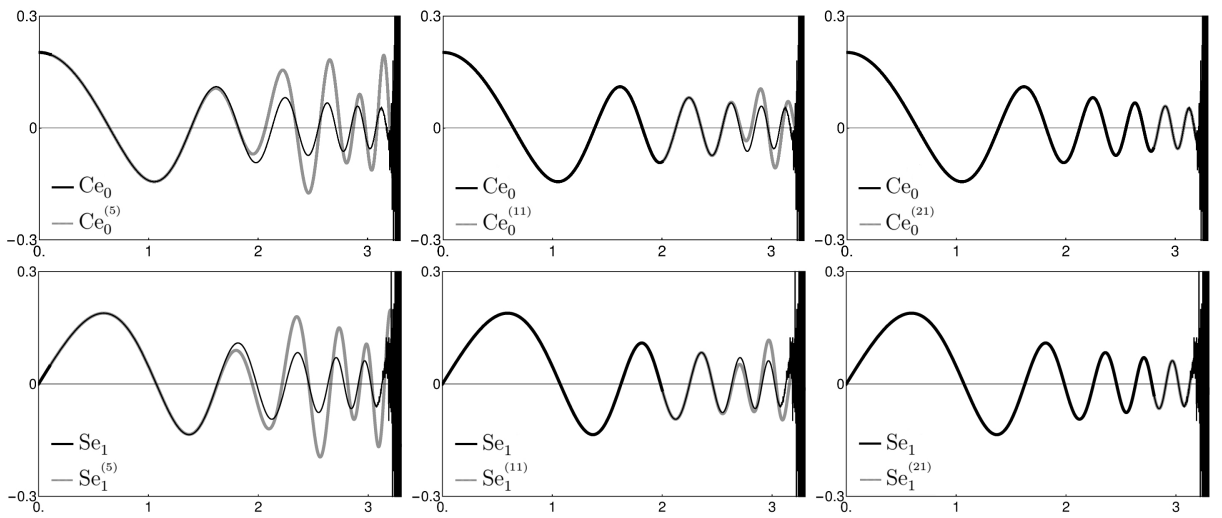

Figure 5: Discrete vs. continuous 'radial' Mathieu functions in the interval $0 \leq \varrho<3.3$, for $N \in\{5,11,21\}$ and here for $q=2$. The 'discrete' functions $\mathrm{Ce}_{n}^{(N)}(\varrho, q)$ and $\operatorname{Se}_{n}^{(N)}(\varrho, q)$ with the (continuous) argument $\varrho$ (gray line), is compared with the 'continuous' functions $\mathrm{Ce}_{n}(\varrho, q)$ and $\mathrm{Se}_{n}(\varrho, q)$ (thin black line). As before, where both coincide within $10^{-16}$ they are replaced by a thick black line. The radial Mathieu functions, when computed with the commercial Mathematica algorithm, oscillate wildly after an upper value that decreases with increasing values of $q$.

the terms in the sum. It is different from zero for $0<\psi_{k}<\frac{1}{2} \pi$ however, so if we choose $\psi_{k}=\frac{1}{4} \pi$, where both summands in the exponent appear as $1 / \sqrt{ } 2$, we can write

$$
\begin{aligned}
\operatorname{Se}_{2 n+2}^{(N)}(\varrho, q)= & K_{2 n+2}^{s} \sum_{m=0}^{N-1} \operatorname{se}_{2 n+2}^{(N)}\left(\psi_{m}, q\right) \\
& \times \exp \left[\mathrm{i} \sqrt{2 q}\left(\cosh \varrho \cos \psi_{m}+\sinh \varrho \sin \psi_{m}\right)\right]
\end{aligned}
$$

For the corresponding continuous case, we could not find a corresponding integral in [7, §6.92], so we cannot give a closed expression for the coefficient $K_{2 n+2}^{s}$ in 38 . The lack of a similar plane-wave integral expression for the continuous Mathieu functions $\operatorname{Se}_{2 n+2}(\varrho, q)$ has been noted also in Ref. [12] without explanation. However, we have checked numerically that the simile of the discrete to continuous functions approximation provided by

$$
\operatorname{Se}_{2 n+2}^{(N)}(\varrho, q) \simeq-\mathrm{i} \operatorname{se}_{2 n+2}(\mathrm{i} \varrho, q)=\operatorname{Se}_{2 n+2}(\varrho, q),
$$

which is an equality for continuous functions, $c f$. [7, 8.611.4, 8.631.4]. For $0<\varrho<2$ the difference in 39 less than $10^{-14}$. We should note that generally the discrete 'radial' and 'angular' Mathieu functions for pure imaginary 
arguments are not related to similar equalities of their continuous integral expressions, because the summation definitions in (26) involve hyperbolic functions. In particular, say,

$$
\operatorname{Se}_{2 n+p+1}^{(N)}(\varrho, q) \neq-\operatorname{ise}_{2 n+p+1}^{(N)}(\mathrm{i} \varrho, q)=\sum_{m=0}^{N-1} b_{2 m+p+1}^{2 n+p+1} \sinh [(2 m+p+1) \varrho] .
$$

\section{Concluding remarks}

The expansion of Helmholtz plane waves in series of radial Bessel and angular trigonometric functions has its discrete analogue in Eq. (16), which tells us that the wavefield due to a finite number $N$ of plane waves at equidistant direction angles can be expanded in discrete Bessel radial functions and corresponding trigonometric angular functions. A similar statement will hold when the wavefield is expanded in discrete Mathieu functions with the phases determined by the points on an ellipse as depicted in Fig. 2. Conceivably such fields can be produced in resonant two-dimensional micro-cavities fed by a number of activation channels.

We recognize that the full treatment and exploration of properties for the discrete Bessel and Mathieu function presented here is not exhaustive, but that it should be sufficient to indicate that the approximation method consisting in the replacement of a continuous closed subgroup of the symmetry group of a partial differential equation by a finite discrete group is definitely of interest. In the present case of two dimensions, the orthogonal group was reduced to the dihedral group. In three dimensions, the symmetry Euclidean symmetry group could reduce its three-dimensional rotation subgroup by any of its polyhedral subgroups, whose functions may serve to describe wavefields with a corresponding subset of wave propagation directions. Here we have presented a set of exact relations, others whose approximation closeness was estimated through numerical computation, and others that have been only suggested by that approach, and for which we expect to present further results from ongoing work.

\section{Acknowledgments}

We thank the support of the Universidad Nacional Autónoma de México through the PAPIIT-DGAPA project AG100120 Óptica Matemática. 


\section{References}

[1] W. Miller Jr., Symmetry and Separation of Variables, Encyclopedia of Mathematics, Vol. 4 (Cambridge Univerity Press, 1984).

[2] G. Biagetti, P. Crippa, L. Falaschetti, and C. Turchetti, Discrete Bessel functions for representing the Class of Finite Duration Decaying Sequences, European Signal Analysis Conference, pp. 2126-2130 (Budapest, 2016).

[3] K. Uriostegui and K.B. Wolf, Discrete Bessel functions and transform, (submitted) arXiv:2005.06076 [math-ph]

[4] R.H. Boyer, Discrete Bessel functions, J. Math. Anal. Appl. 2, 509-524 (1961).

[5] M. Bohner and T. Cuchta, The Bessel difference equation, Proc. Amer. Math. Soc. 145, 1567-1580 (2017).

[6] A. Slavík, Discrete Bessel functions and partial differential equations, $J$. Diff. Eqs. Applics. DOI:10.1080/10236198.2017.141610 (2017).

[7] I.S. Gradshteyn and I.M. Ryzhik, Tables of Integrals, Series and Products, A. Jeffrey and D. Zwillinger Eds, (Academic Press, 2007).

[8] G.N. Watson, Theory of Bessel Functions (Cambridge University Press, 1922).

[9] A. Erdélyi et al., Higher Transcendental Functions (Based on notes by H. Bateman) Vol. 2 (McGraw-Hill, New York, 1953).

[10] P. Winternitz, K.B. Wolf, G.S. Pogosyan, and A.N. Sissakian, Graf's addition theorem obtained from $\mathrm{SO}(3)$ contraction, Theor. Mat. Phys. 129, 1501-1503 (2001).

[11] N.W. McLachlan, Theory and Application of Mathieu Functions (Oxford University Press, 1947).

[12] L. Chaos-Cador and E. Ley-Koo, Mathieu functions, matrix evaluation, and generating functions, Rev. Mex. Fis. 48, 67-75 (2002). 